\title{
Adaptive Log Compression for Massive Log Data
}

\author{
Robert Christensen \\ Feifei Li \\ robertc@eng.utah.edu, lifeifei@cs.utah.edu, School of Computing, University of Utah
}

\begin{abstract}
We present a novel adaptive log compression scheme. Results show $30 \%$ improvement on compression ratios over existing approaches.
\end{abstract}

\section{Categories and Subject Descriptors}

H.2.4 [Information Systems]: Database Management - Systems

\section{Keywords}

Log compression, adaptive log compression, log data management

\section{PROBLEM STATEMENT}

Log data is ubiquitous and humongous. The standard log compression method is to compress the entire log data together.

But in practice log entries are often heterogeneous, with varying patterns over time. They also have strong temporal locality. Thus, a better approach is to adaptively distribute entries to different buckets, and compress buckets separately in parallel. Formally,

Definition 1 (Adaptive Log Compression) For a $\log$ data $D$ with $n \log$ entries $e_{1}, \ldots, e_{n}$ (sorted on arrival timestamps), a budget $g$, produce $g$ disjoint $\log$ buckets $B_{1}, \ldots, B_{g}$, such that $\forall i \in[1, n]$, $\exists j \in[1, g], e_{i} \in B_{j}$, and $\forall x, y \in[1, g], B_{x} \cap B_{y}=\emptyset$. Each bucket also stores sorted $\log$ entries. Given any compression method $Z$, $Z(D)$ is the compressed output with size (in bytes) $|Z(D)|$. The objective is to maximize $|Z(D)|-\sum_{j=1}^{g}\left|Z\left(B_{j}\right)\right|$.

\section{TECHNICAL APPROACH}

Consider a snippet from a real log data in Figure1 1 To minimize heterogeneity in a log bucket, ideally, log entries in green solid box should be partitioned into one log bucket, and entries in red dashed box will be in another bucket. The challenge is to achieve adaptive distribution online to get homogeneous buckets.

We impose a sliding window $w_{i}$ of size $m$ on bucket $B_{i}$, that keeps track of most recent $m$ entries in $B_{i}$. The $j$ th entry in $w_{i}$ is $e_{i, j}\left(e_{i, 1}\right.$ being the most recent). For each entry, we construct a signature with a mapping function $\sigma$, i.e., $\sigma_{i, j}=\sigma\left(e_{i, j}\right)$. Assume a similarity function $\operatorname{sim}:\left(\sigma_{1}, \sigma_{2}\right) \rightarrow[0,1]$ which gives a similarity score for any two signatures. For an incoming log entry $e_{\text {next }}$, we define the score of $e_{\text {next }}$ on the $i$ th bucket $B_{i}$ as follows:

$$
s\left(e_{\text {next }}, i\right)=\operatorname{avg}\left\{\operatorname{sim}\left(\sigma\left(e_{\text {next }}\right), \sigma_{i, j}\right), \text { for } j \in[1, m]\right\},
$$

i.e., the average similarity between $e_{\text {next }}$ 's signature and any signature of the $m$ entries in $w_{i}$. Our adaptive scheme sends $e_{\text {next }}$ to bucket $B_{j}$ with the maximum score, i.e, $j=\operatorname{argmax}_{i \in[1, g]} s\left(e_{\text {next }}, i\right)$.

Copyright is held by the author/owner(s).

SIGMOD'13, June 22-27, 2013, New York, New York, USA.

ACM 978-1-4503-2037-5/13/06.

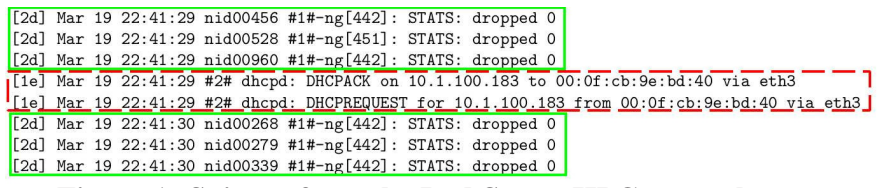

Figure 1: Snippet from the Red Storm HPC server log.

When all entries have been processed, we compress $B_{1}, \ldots, B_{g}$ independently in parallel. We base our construction of $\sigma$ by viewing each $\log$ entry as a set of elements (e.g., $q$-grams). We then develop a $\log$ signature $\sigma$ based on the $k$-minimum value synopses [1]. Different similarity functions sim can be used; we report only the one that is a variant of the Jaccard similarity.

\section{MAIN RESULTS}

Compressing the entire log together is dubbed centralized. Two naive bucketization methods, round robin and segmentation, were also tested. The former distributes entries to different buckets in a round robin fashion; the latter divides $D$ into $g$ disjoint but contiguous segments with same number of entries.

We show the results using gzip on an Apache web server log. It has 26 million entries, totaled 8GB. Other compression methods and datasets were also tested, giving similar results.

We use centralized as a reference point which has a compressed size of 533MB using gzip. The output size of any method is shown as a ratio to the output size of centralized in Figure2 For adaptive, round robin, and segmentation methods, the default bucket budget $g$ is 32; for adaptive, the default sliding window size $m$ is 10 .

Figure 2 shows up to $30 \%$ improvement in compression ratios achieved by adaptive over centralized, using small $g$ and $m$ values. It also shows the ineffectiveness of naive bucketizations. Lastly, because adaptive is a streaming algorithm, and can distribute entries and compress buckets independently in parallel, its overall running time is comparable to that in centralized (omitted for space).

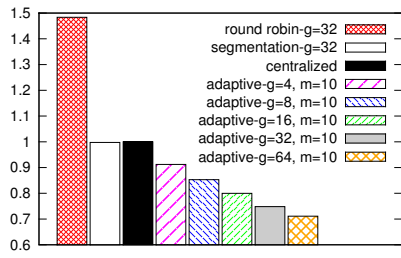

(a) Vary bucket budget $g$.

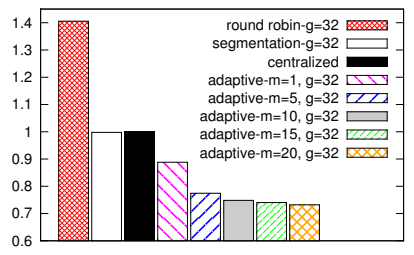

(b) Vary sliding window size $m$.
Figure 2: Effect of bucket budget and history depth.

\section{CONCLUDING REMARKS}

An interesting challenge is to improve adaptive to learn the best values for $g$ and $m$ online and adjust them dynamically over time.

\section{REFERENCES}

[1] K. S. Beyer, P. J. Haas, B. Reinwald, Y. Sismanis, and R. Gemulla. On synopses for distinct-value estimation under multiset operations. In SIGMOD, 2007. 